\title{
Meta-análisis: La monoterapia con estatinas es eficaz para la prevención de accidentes cerebrovasculares
}

Reductase inhibitor monotherapy and stroke prevention. Crouse III J, Byington R, Hoen $\mathrm{H}$ et al.

Arch Intern Med. 1997; 157: 1305-1310

Objetivo

Meta-análisis para determinar los efectos de la reducción de la colesterolemia con estatinas sobre la incidencia de Accidente $\mathrm{Ce}$ rebrovascular (ACV).

\section{Fuentes de Datos}

Búsqueda en MEDLINE (hasta 1995) de todo ensayo clínico con estatinas como monoterapia hipolipemiante.

\section{Selección de Estudios}

Se seleccionaron 12 estudios randomizados que cumplieron los siguientes criterios: 1) utilización de estatinas como monodrogas, 2) registro de eventos cardiovasculares como puntos finales, y 3 ) reporte de la incidencia de ACV. Se incluyeron 4 ensayos de prevención primaria de enfermedad coronaria y 8 de prevención secundaria.

\section{Resultados Principales}

Entre los 19.500 pacientes incluidos se reportaron $430 \mathrm{ACV}$, 104 en estudios de prevención primaria y 326 en los de prevención secundaria. El seguimiento promedio fue de 3.1 años. Analizando en conjunto los estudios de prevención primaria y secundaria, se registraron $182 \mathrm{ACV}$ en el grupo con fármacos y 248 en el grupo placebo, lo que significa una disminución del riesgo de ACV del 27\% o RR 0.73 (IC 95\% 0.60-0.89).

El análisis por separado de los ensayos de prevención primaria y secundaria mostró los siguientes resultados:

\begin{tabular}{|c|c|c|c|c|}
\hline \multirow[t]{2}{*}{ 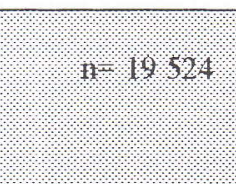 } & \multicolumn{2}{|c|}{ 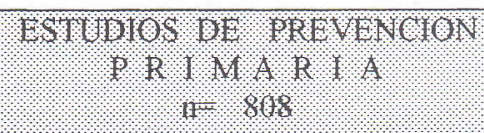 } & \multicolumn{2}{|c|}{ 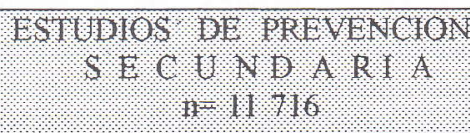 } \\
\hline & ESTATINAS & PLACEBO & ESTATINAS & PLACEBO \\
\hline Nimmero de $\Lambda$ C X & 48 & 56 & 134 & 192 \\
\hline RR ( $1(105 \%$. & \multicolumn{2}{|c|}{$0.85(0.58-1.27)$} & \multicolumn{2}{|c|}{$0.69 * *(0.55-0.87)$} \\
\hline RRA II IICY & \multicolumn{2}{|c|}{$15 \%$} & \multicolumn{2}{|c|}{$31 \% * *$} \\
\hline
\end{tabular}

Referencias: ** Significativo $(\mathrm{P}<0.005)$, RRR: Reducción de Riesgo Relativo*, RR: Riesgo Relativo*

En los 8 estudios de prevención secundaria hubo 673 eventos coronarios en el grupo estatinas y 954 en el grupo placebo, lo que implica una disminución del riesgo de cas un $30 \%$ o RR 0.70 que es muy similar al RR para ACV $(0.69$ -ver tabla-)

\section{Comentario}

Si bien existe clara evidencia sobre el descenso del colesterol y la disminución del riesgo coronario, la relación con el ACV y la mortalidad general es controvertida. Un meta-análisis de 45 ensayos que reunió $13.000 \mathrm{ACV}$ entre 450.000 pacientes, no encontró asociación alguna (1). De hecho un meta-análisis previo concluyó que la disminución de la colesterolemia no disminuye la morbimortalidad por ACV y que el clofibrate incrementaría el riesgo de ACV fatal, especialmente hemorrágico (2). Por otra parte se relacionó el tratamiento hipolipemiante con el aumento de mortaliciad por causas no cardiovasculares como muerte violenta y cáncer (3). Estos estudios no evaluaban al riesgo de ACV como resultado principal. Cabe entonces suponer que los efectos observados podrían estar dados por diferentes motivos: falta de poder de los estudios, efectos indeseables de las drogas ensayadas, efectos farmacodinámicos específicos. Un primer indicio que insinuaba la asociación entre colesterol y ACV lo reveló un meta-análisis de 4 estudios con pravastatina que mostró una disminución no significativa $(\mathrm{P}=0.054)$ del riesgo de $\mathrm{ACV}$ de un $60 \%$ (4). Los dos presentes trabajos analizaron los principales ensayos de la última década que evaluaron a las estatinas como monoterapia. Ambos analizaron riesgo de ACV, y el segundo evaluó además mortalidad general. Se observó una reducción del riesgo relativo* de ACV de $27-29 \%$. Cuando analizamos separadamente estudios de prevención primaria y secundaria de coronariopatía, encontramos en los primeros una disminución no significativa del 15 al

\section{Conclusiones}

El descenso de los niveles de colesterol con estatinas disminuye el riesgo de $\mathrm{ACV}$, especialmente en pacientes con enfermedad coronaria.

Fuente de Financiamiento: No referida
$20 \%$ y en los segundos una disminución significativa mavor del $30 \%$ Esto podría deberse a la menor incidencia de ACV en los pacientes no coronarios. Es posible que aumentando el número de pacientes las diferencias sean significativas; sin embargo el número necesario para tratar* sería muy grande, tornando menos favorable la relación costo-efectividad de la intervención. Otro punto es si la disminución del riesgo de ACV es exclusivamente a expensas de los isquémicos (más relacionados fisiopatológicamente con el colesterol). Lamentablemente, los subtipos de eventos cerebrovasculares no están discriminados en los estudios analizados, pero en el caso que los hemorrágicos no diminuyeran, se diluiría el efecto beneficioso sobre los isquémicos con lo cual el beneficio sería aún mayor. Es más. en el peor de los casos en que el riesgo de ACV hemorrágico aumentara con niveles bajos de colesterol como insinuara algún estudio previo (5), el efecto neto sobre la mortalidad por ACV observado en estos estudios es aún favorable. En cuanto a la mortalidad general ve mos que disminuye tanto en el grupo de prevención primaria ( $26 \%$ como secundaria $(21 \%)$ sin cambios sobre la mortalidad no cardiovascular. Estos resultados beneficiosos con estatinas podrían deberse a varias causas: mayor descenso del colesterol frente a otras drogas (20\% vs. $10 \%$ ) y de LDL (30-40\% vs. $10-12 \%$ ); efectos adicionales específicos (restauración endotelial, estabilización de placa ateromatosa): mínimos efectos adversos. Además es factible que part: de la disminución del riesgo de ACV sea secundario a la significa- 